Inarejos Muñoz, J.A. (2017). El uso de fuentes históricas y bases documentales en la formación del profesorado de Educación Infantil y Primaria. Revista Electrónica Interuniversitaria de Formación del Profesorado, 20(2), 157-166.

\title{
El uso de fuentes históricas y bases documentales en la formación del profesorado de Educación Infantil y Primaria
}

\author{
Juan Antonio Inarejos Muñoz \\ Universidad de Extremadura
}

\section{Resumen}

En este artículo se desarrolla una experiencia metodológica innovadora con alumnos de los grados de Educación Infantil y Educación Primaria centrada en el manejo de fuentes primarias y una base documental en línea. El objetivo de este trabajo persigue familiarizar a los estudiantes con la metodología propia del conocimiento histórico para desarrollar nuevas experiencias educativas donde el trabajo del alumno pasa a un primer plano. A través de la selección de familiares o de personajes aleatorios, se rastrearon en la base documental https://familysearch.org/ los nacimientos, matrimonios y defunciones de sus antepasados. Una vez extraída esta información se elaboraron árboles genealógicos de cada una de las familias escogidas utilizando algunas de las aplicaciones web sugeridas.

\section{Palabras clave}

Enseñanza de la Historia; fuentes primarias; bases documentales; investigación histórica.

\section{Contacto:}

Juan Antonio Inarejos Muñoz, profesor Contratado Doctor, Facultad de Formación del Profesorado (Cáceres), Avenida de la universidad, s/n, 10071, Teléfono 927257973, juaninarejos@unex.es, Universidad de Extremadura.

Este artículo constituye un resultado del proyecto de investigación "La evaluación de las competencias y el desarrollo de capacidades cognitivas sobre Historia en Educación Secundaria", EDU2015-65621-C3-2-R, subvencionado por el Ministerio de Economía y Competitividad. 


\title{
The use of historical sources and documentary bases in the training of teachers of Early Childhood and Primary Education
}

\begin{abstract}
The aim of this paper is to show an innovative approach to students of the grade of infant and primary education by working with primary sources and the development of active teaching methods. This practice is organized around strategies of research, combining work with the project method, the case study or the Problem-based learning to promote the acquisition of skills, and abilities in students. This attempted to develop a learning experience based on the development of historical research and history teaching. The primary sources used were the religious information on births, marriages and deaths housed in a digital documentary base. Subsequently, the extracted information was treated with multimedia applications.
\end{abstract}

\section{Key words}

History teaching; primary sources; digital documentary bases; historical research.

\section{Introducción}

En este artículo se desarrolla una experiencia metodológica innovadora con alumnos de los grados de Educación Infantil y Educación Primaria centrada en el manejo de fuentes primarias y una base documental digital. El objetivo de este trabajo persigue fomentar en los futuros maestros habilidades, capacidades y competencias a partir del análisis de fuentes históricas primarias obtenidas de una base documental en línea fomentando las estrategias de investigación y el trabajo cooperativo. En último término, se busca familiarizar a los estudiantes con la metodología propia del conocimiento histórico para desarrollar experiencias educativas innovadoras donde el trabajo del alumno pasa a un primer plano. A través de la selección de familiares o de personajes aleatorios, se rastrearon en la base documental https://familysearch.org/ ${ }^{1}$ los nacimientos, matrimonios y defunciones de sus antepasados. Una vez extraída esta información se elaboraron árboles genealógicos de cada una de las familias escogidas utilizando algunas de las aplicaciones web existentes sugeridas por el profesor.

A través de esta experiencia se pretenden desplegar e implementar destrezas relacionadas con la clasificación de fuentes, la utilización de fuentes primarias en el proceso de enseñanza-aprendizaje del tiempo histórico, la búsqueda de información, el tratamiento de información obtenida con herramientas informáticas y el uso de bases de datos documentales virtuales. Este proyecto a su vez también permite trabajar de forma indirecta con la familia, uno de los principales ámbitos de socialización del alumnado, y la evolución y transformaciones que ha sufrido a lo largo de distintas etapas históricas.

\footnotetext{
${ }^{1}$ Comencé a utilizar esta base de datos a partir de la investigación sobre la biografía sobre un político y periodista del siglo XIX, Francisco Córdoba y López. Distintas fuentes secundarias aportaban datos contradictorios sobre su lugar de nacimiento, un dato fundamental para comprender una de sus principales obras censurando el caciquismo. La consulta de la partida de nacimiento original en esta base documental me sacó de dudas. Constituye un buen ejemplo de la trascendencia de la investigación histórica para los docentes de didáctica de la Historia y la importancia de la formación disciplinar.
} 


\section{La enseñanza de la historia a través de la investigación con fuentes primarias en bases documentales en línea}

El potencial didáctico que entraña la introducción en las aulas de Infantil y de Primaria el trabajo con fuentes históricas y la metodología propia del conocimiento histórico resulta innegable. Pero también resulta absolutamente necesario para proponer en las aulas una enseñanza de la Historia basada en el desarrollo de habilidades y el pensamiento crítico que huya de una visión tradicional basada en la clase magistral y la memorización de contenidos. En este nuevo enfoque la utilización de las nuevas tecnologías en la enseñanza de la Historia constituye un recurso fundamental, desde las bases documentales en red hasta las aplicaciones informáticas que permiten trabajar en las aulas con la información extraída. No obstante, en estas edades tan tempranas la implementación de estas metodologías entraña una considerable complejidad (Tonda, 2001; Pastor, Alonso, Luna, Jiménez, Martín, Santisteban, 2015; Aranda, 2016). En relación al alumnado del Grado de Educación Infantil, y según recoge la normativa educativa, durante esta etapa debe conocer fundamentalmente las distintas tipologías y características que se pueden establecer en relación al concepto de tiempo: el tiempo astronómico o físico -al que mayor atención se presta en estas edades tan tempranas- y el tiempo humano, colectivo o social. Pero también, una vez los niños son capaces de comprender las dimensiones de la temporalidad (duración, sucesión, simultaneidad o ritmo), los postreros conceptos de tiempo cronológico y tiempo histórico que pueden comenzar a tratarse incipientemente al final de esta etapa aunque se trabajen de forma sistemática más tarde, en Educación Primaria (Trepat, 2002, 2011; Chacón, 2009; Hernández Cervantes, 2014).

En suma, durante el primer ciclo de Educación Infantil se insiste en el trabajo con horarios, calendarios e instrumentos de medición del tiempo en el día a día para familiarizar a los niños con los distintos elementos del tiempo físico y civil (segundos, minutos, horas, días, semanas o estaciones), pero también resulta pertinente incluir actividades más complejas basadas en las primeras tomas de contacto con las fuentes históricas a finales del segundo ciclo de Educación Infantil (Trigueros, Arias, Miralles, Molina, Rodríguez, 2013: 90-94). En este sentido, tienen un gran potencial didáctico actividades como el objeto del día (Aranda, 2003), el baúl o caja del tiempo (Miralles, Molina, 2011), la caja genealógica (Cuenca, Estepa, 2005), las historias de vida (Bilbao, Pérez, Ezkurdia, 2014), las dramatizaciones de personajes, etapas y hechos del pasado (Miralles, Rivero, 2012) o las cajas de vida (Anguita, 2012). Todas estas actividades, aunque centradas en el análisis de objetos antiguos y las vivencias de los alumnos o sus familias, también permiten introducir una primera aproximación con las fuentes históricas que tan sugerentes resultados y potencialidades aporta según han apuntado distintos autores (Haydn, Arthur y Hunt, 1997; Cooper, 2002; Pagès y Santisteban, 2010; Feliú, Hernàndez Cardona, 2011; Prieto, Gómez, Miralles, 2013). En este sentido, resulta perentorio que los futuros docentes también conozcan algunos aspectos básicos -pero claves- de la metodología de la Historia, como la clasificación y crítica de las fuentes, para rellenar el vacío de la formación disciplinar que sí poseen los historiadores. Baste recordar, entre otros, los trabajos de Alía (2008), Moradiellos (2013) o Domínguez (2015), donde el trabajo con fuentes históricas se presenta como un ejercicio enriquecedor en la formación de los futuros maestros para acercarlos a los rudimentos del trabajo del historiador, imprescindible para desplegar en las aulas la metodología de la Historia. Como ha defendido Joaquín Prats: 
"¿Qué principios son imprescindibles para un correcto proceso de enseñanza aprendizaje de la historia en las etapas escolares? Desde mi posición en el debate didáctico, la respuesta es clara y contundente: la enseñanza de la historia debe consistir en la simulación de la actividad del historiador y el aprendizaje en la construcción de conceptos, familiarizando al alumnado a: formular hipótesis; aprender a clasificar fuentes históricas; aprender a analizar las fuentes; aprender a analizar la credibilidad de las fuentes, el aprendizaje de la causalidad y, por último, el cómo iniciarse en la explicación histórica." $(2010,15)$.

La clasificación de fuentes históricas constituye un ejercicio fundamental para iniciar a los futuros docentes de Educación Infantil y Primaria en el trabajo del historiador. Las visitas guiadas a los archivos y las actividades planteadas por los gabinetes didácticos en torno a la conservación, tipología y clasificación de fuentes entrañan un potencial difícil de superar. No obstante, las enormes posibilidades que ofrecen las bases documentales en línea permiten desarrollar en el aula unidades didácticas y actividades muy sugerentes y enriquecedoras para familiarizar a los alumnos con los mimbres que utilizan los historiadores para confeccionar el conocimiento histórico. En último término, se pretende fomentar el trabajo autónomo, la reflexión y el espíritu crítico en los alumnos a través del planteamiento de problemas de investigación relacionados con las fuentes que no sean excesivamente simples pero que tampoco entrañen una gran complejidad. El papel del profesor dentro de estas estrategias está vinculado a orientar a los alumnos sobre las bases documentales donde pueden acceder a las fuentes, enseñar las herramientas necesarias para poder sistematizar la información recibida y azuzar el interés investigador de los alumnos para que sean capaces de lanzar hipótesis e intentar contrastarlas.

\section{Planteamiento didáctico}

La irrupción de las tecnologías de la información y la comunicación en las últimas décadas también han revolucionado el acceso, la localización y el tratamiento de la documentación de muchos archivos y ha multiplicado las posibilidades de investigación de los historiadores. Pero también han disparado las posibilidades para implementar en las aulas experiencias didácticas innovadoras relacionadas con el acceso, clasificación y uso didáctico de fuentes históricas a través del acceso virtual. Baste recordar las posibilidades que brindan las colecciones de prensa, libros o documentos digitalizadas de la Biblioteca Nacional (www.bne.es), el Portal PARES (http://pares.mcu.es/) destinado a la difusión en Internet del Patrimonio Histórico Documental Español conservado en su red de centros, las colecciones digitales albergadas en la Biblioteca Tomás Navarro Tomás del Centro de Ciencias Humanas y Sociales del CSIC (http://biblioteca.cchs.csic.es) o la Biblioteca Virtual Cervantes (http://www.cervantesvirtual.com/).

La innegable relevancia y valor histórico de las fuentes albergadas en estos repositorios, unidas a las estrategias docentes desplegadas por el profesor, puede despertar el interés de los alumnos. Pero esta motivación se multiplica exponencialmente si las fuentes históricas están relacionadas o vinculadas con sus parientes y antepasados, en definitiva, con la historia cercana (Prieto, Gómez, Miralles, 2013: 8). La selección de fuentes sencillas que no entrañen una excesiva complejidad permite combinar el interés de los alumnos y las evidentes carencias en la formación disciplinar como historiadores de los maestros, particularmente en el conocimiento paleográfico. No obstante, los libros de bautismo, matrimonio o defunción digitalizados de una parte significativa de la población española de los últimos siglos permiten conjugar ambos elementos, al tratarse de fuentes que no 
revisten una gran complejidad que hacen referencia a los antepasados de los alumnos. Además, permiten acercarse a las primeras fases de la metodología histórica vinculadas a la búsqueda y selección de las fuentes históricas, fundamental para que el alumno no sea un mero receptor de contenidos históricos. También permiten combinar el trabajo independiente -por la facilidad de acceso a las fuentes- con el trabajo cooperativo en grupo. Finalmente, desde el punto de vista de los sujetos de la Historia (De la Montaña, García, Hernández, 2015), supone una innegable innovación al desplazar el tradicional foco de reyes, militares y grandes hombres de Estado para acercarse a los desconocidos "Fulanos y Menganos" de los que hablaba en sus agudas novelas Benito Pérez Galdós (Inarejos, 2013).

La experiencia educativa que se ha llevado a cabo perseguía varios horizontes. El trabajo en las aulas con estas fuentes primarias permite analizar cuestiones paralelas fundamentales para otras áreas vinculadas a la Didáctica de las Ciencias Sociales como los cambios que han experimentado los modelos de familia a lo largo de la Historia, las transformaciones demográficas que han sufrido las sociedades, el éxodo rural, la evolución de las creencias religiosas o la aplicación de las nuevas tecnologías a la enseñanza de la Historia. En este sentido, se ha implementado una experiencia didáctica con la base documental disponible en la web https://familysearch.org/. Constituye una de las colecciones más exhaustivas de registros genealógicos e históricos del mundo, recogidos y digitalizados en distintas partes del mundo durante los últimos cien años por una congregación religiosa norteamericana. Aunque esta base de datos pertenece a una institución privada la consulta de los fondos es gratuita, de libre acceso digital, y cumple con los criterios de recopilación, captura de imágenes, indexación y conservación de registros. Es decir, constituye un auténtico filón documental para la investigación histórica pero también para la enseñanza de la Historia a distintos niveles educativos. En esta base figuran digitalizados en línea una cantidad importante de los libros de bautismo, matrimonio o defunción de la población española -y de otros países- de los últimos siglos. Para el caso concreto de España esta base documental recoge información de 46 colecciones documentales entre los años 1251-2013 con 24.552.510 registros indexados y 24.168 .442 imágenes de registros.

Las búsquedas se pueden realizar introduciendo los nombres en el buscador "Registros" por acontecimientos de la vida de una persona como el nacimiento, el matrimonio, la residencia, la defunción o el grado de parentesco con otra persona (aunque este buscador no ofrece grandes resultados porque la información de muchos de los documentos no está volcada en esta base de datos). No obstante, para fomentar las destrezas de indagación y realizar búsquedas precisas es recomendable que las búsquedas se realicen en la pestaña "Investigación por lugar", acotando progresivamente la zona de búsqueda y las fechas (Europa-España-provincia).

\section{Nivel de agrupamiento y cursos que desarrollan la actividad}

Esta práctica se implementó en dos grupos de las asignaturas Didáctica de las Ciencias Sociales del tercer curso del Grado de Educación Infantil y de la asignatura Conocimiento del medio físico, social y cultural del tercer curso del Grado de Educación Primaria (curso 20152016). Esta experiencia integró el trabajo cooperativo en los seminarios establecidos con el trabajo autónomo de los alumnos tras la asignación de funciones individualizadas. Se dividieron ambas clases en grupos de cuatro o cinco integrantes.

\section{Objetivos didácticos}

- Subrayar el papel central que ocupan las fuentes históricas en la metodología de la Historia. 
- Aprender a clasificar e interpretar las fuentes históricas: primarias y secundarias.

- Mostrar la relevancia de las fuentes históricas para corroborar o desechar hipótesis de investigación.

- Familiarizar a los alumnos con el acceso y manejo de bases documentales en red.

- Trabajar las destrezas relacionadas con el tratamiento de la información obtenida en las fuentes históricas con herramientas vinculadas a las tecnologías de la información y la comunicación.

- Evidenciar a través de las fuentes los cambios que ha sufrido la familia, desde el modelo tradicional o nuclear predominante en épocas pasadas a la irrupción de nuevos modelos de familia en la sociedad actual.

- Comparar la evolución de los regímenes demográficos antiguos y modernos.

- Reflexionar sobre la trascendencia histórica de los procesos migratorios vinculados al éxodo rural y los cambios operados en las profesiones.

- Analizar los cambios sociológicos relacionados con la evolución de las creencias religiosas y la utilidad de las fuentes religiosas en épocas pasadas y en el presente.

\section{Contenidos}

- Conceptos temporales (tiempo cronológico-tiempo histórico).

- Fuentes históricas (primarias y secundarias).

- Conceptos sociales (familia, profesiones y entorno social).

- Estudio y comparación de la familia y los índices de población (nacimientos, residencia, matrimonios y mortalidad) entre épocas pasadas y el presente.

- La evolución del peso de la religión en la sociedad española.

- La evolución de la sociedad española.

- Utilización de nuevas tecnologías (bases de datos y aplicaciones multimedia).

\section{Temporización}

Este trabajo se llevó a cabo en las horas de seminarios a lo largo de cuatro semanas del primer trimestre, en el tema consagrado a la Didáctica de la Historia en ambas asignaturas.

\section{Desarrollo del proyecto}

La experiencia se desarrolló en distintas fases:

- En primer lugar se mostró a los alumnos cómo funciona la base de datos: la organización del catálogo, modalidades de búsquedas y el tipo de información que se puede extraer (nacimientos, matrimonios, residencia y defunciones).

- Visualización de fuentes pertenecientes a distintos siglos para que los alumnos observen la complejidad de trabajar con fuentes antiguas.

- Lectura de distintos documentos de los siglos XIX y XX para habituar a los alumnos con el tipo de letra y de fuentes que puede encontrar en esta base de datos. También se mostraron documentos de la Edad Medida y de la Edad Moderna para que los alumnos observasen la progresiva complejidad que entraña analizar o transcribir fuentes antiguas.

- Selección por cada uno de los grupos de un familiar o de una persona al azar para reconstruir su árbol genealógico e información complementaria como su oficio, 
matrimonio, lugar de residencia o familiares. Se recomendó que las búsquedas no se remontasen excesivamente más allá de cuatro o cinco generaciones por los problemas que podía entrañar la lectura de documentos muy antiguos sin conocimientos paleográficos. Las búsquedas podían realizarse en grupo o distribuyéndose entre sus integrantes las distintas ramas familiares.

- Una vez obtenida la información, puesta en común de los integrantes del grupo y volcado de los datos en un árbol genealógico (Molina, 2011), elaborado con alguna de las múltiples aplicaciones web existentes como www.genoom.com, www.gnoom.com o www.myheritage.es, que complementan y enriquecen el tradicional árbol genealógico elaborado con murales y expuesto en las paredes de las aulas. Además, plantea la posibilidad de incorporar documentos originales descargados de la base documental para explicitar la importancia de las evidencias empíricas para el historiador a la hora de elaborar sus hipótesis y el conocimiento histórico.

- Es recomendable que la muestra de los distintos grupos de la clase englobe distintos tipos de familias para mostrar la diversidad existente en la sociedad actual y los sensibles cambios demográficos, espaciales, económicos, sociales y religiosos operados respecto a épocas pasadas gracias a la información aportada por los alumnos.

Metodología

- Desde el punto de vista metodológico se ha combinado el trabajo personal de los alumnos con el trabajo en grupo incidiendo en metodologías activas basadas en procedimientos, actitudes y desarrollo de destrezas vinculadas a la investigación.

- Desde el punto de vista del docente, el profesor se erige en un guía que explica y facilita las técnicas y la metodología para la investigación pero no actúa como un mero transmisor de contenidos.

- Análisis de casos y resolución de problemas. Presentación de diferentes casos y exposición de recursos para resolverlos con actividades dirigidas a introducir nociones teóricas y a aplicar competencias de las materias incluidas a partir de supuestos prácticos (como la búsqueda de los antepasados para realizar una solicitud de nacionalidad).

\section{Discusión y conclusiones}

Los resultados obtenidos con esta experiencia educativa han permitido alcanzar con solvencia los objetivos propuestos inicialmente. En primer lugar, trabajando en el aula con la metodología de la Historia y el papel que desempeñan las fuentes en la elaboración del conocimiento histórico. El análisis de fuentes históricas de distintas etapas ha permitido aquilatar conceptos temporales fundamentales para estas fases educativas como son el tiempo cronológico y tiempo histórico. A su vez, el acceso y manejo de fuentes primarias ha permitido explicitar las diferencias que albergan con las fuentes secundarias y su relevancia para corroborar o desechar hipótesis de investigación. El acceso abierto de esta plataforma ha favorecido familiarizar a los alumnos con el manejo de bases documentales en red y trabajar las destrezas relacionadas con el tratamiento de la información obtenida en las fuentes históricas con herramientas vinculadas a las tecnologías de la información y la comunicación.

Desde el punto de vista de los contenidos los alumnos han podido corroborar de primera mano los cambios que ha sufrido la familia, desde el modelo tradicional o nuclear predominante en épocas pasadas a la irrupción de nuevos modelos de familia en la 
sociedad actual. Por su parte, la información relacionada con la natalidad, la mortalidad y los lugares de residencia ha permitido comparar la evolución de los regímenes demográficos antiguos y modernos y reflexionar sobre la trascendencia histórica de los procesos migratorios vinculados al éxodo rural y los cambios operados en las profesiones del entorno social. En el plano religioso, estas fuentes también han posibilitado reflexionar sobre los cambios sociológicos relacionados con la evolución de las creencias religiosas y la utilidad de las fuentes religiosas para conocer el comportamiento de la población en épocas pasadas y en el presente.

Desde el punto de vista competencial con esta experiencia se han trabajado numerosas competencias básicas, transversales y específicas de las memorias verificadas de ambos grados. Los alumnos han desarrollado la competencia vinculada a la transmisión de información e ideas y las habilidades de aprendizaje necesarias para emprender estudios posteriores con un alto grado de autonomía, pertrechados de las nuevas tecnologías de la información como instrumento de trabajo intelectual y como elemento esencial para informarse, aprender y comunicarse. Además, han elaborado materiales didácticos en soporte digital para el desarrollo del currículo de Educación Infantil y Primaria. Desde el punto de vista conceptual han adquirido conocimientos sobre la evolución de las costumbres y de las creencias a lo largo de la historia que han favorecido la comprensión de los principios básicos de una de las disciplinas vertebradoras de las ciencias sociales.

Los problemas que se han presentado a la hora de trabajar con esta base de datos han sido fácilmente sorteables. Hay alumnos que no han podido encontrar a familiares porque las fuentes han desaparecido o no han podido ser digitalizadas. No obstante, han podido realizar este ejercicio de investigación con personajes aleatorios, incluso de otros países. En el extremo opuesto, el interés que despierta la historia cercana o familiar llevó a algunos grupos a ampliar la búsqueda más allá de los generaciones solicitadas o complementando con pequeñas historias de vida, objetos personales, fotografías o entrevistas de las generaciones más cercanas. Finalmente, y en relación con los procedimientos de evaluación, esta actividad ha permitido calibrar una serie de procedimientos, destrezas y actitudes con una perspectiva metodológica alejada de las tradicionales pruebas finales centradas en simples ejercicios memorísticos.

\section{Referencias}

Alía Miranda, F. (2008). Técnicas de investigación para historiadores: las fuentes de la historia. Madrid: Síntesis.

Anguita, M. (2012). Cajas de vida: paisajes que nos narran. Cuadernos de pedagogía, 422 (Abril), 34-38.

Aranda Hernando, A. Ma (2016). Didáctica de las ciencias sociales en Educación Infantil. Madrid, Síntesis.

Aranda Hernando, A. $M^{a}$ (2003). Didáctica del conocimiento del medio social y cultural en Educación Infantil. Madrid: Ed. Síntesis.

Bilbao B., Pérez, K. y Ezkurdia, M. G. (2014). "La investigación biográfica en la formación inicial de magisterio: las historias de vida como temática del trabajo fin de grado de educación infantil y primaria." Tendencias pedagógicas, 24, pp. 85-98. 
Chacón, A. (2009). Propuesta pedagógica y didáctica para la construcción del pensamiento histórico a partir del pensamiento narrativo en niños y niñas de entre 5 y 7 años. Investigación en educación pedagógica y formación docente. II Congreso Internacional. VII Seminario Nacional. Bogotá: Universidad Pedagógica de Colombia, 231-239.

Cooper, H. (2002). Didáctica de la historia en la Educación Infantil y Primaria. Madrid: Ed. Morata.

Cuenca, J. M. y Estepa, J. (2005). La caja genealógica: Fuentes y tiempo histórico en Educación Infantil. Una propuesta para trabajar con maestros en formación inicial. Quaderns Digitals, 37.

De la Montaña, J. L., García, C. M. y Hernández, A. M. y (eds.) (2015). Una enseñanza de las ciencias sociales para el futuro: Recursos para trabajar la invisibilidad de personas, lugares y temáticas. Cáceres: Universidad de Extremadura: Asociación Universitaria de Profesores de Didáctica de las Ciencias Sociales.

Domínguez, J. (2015). Pensamiento histórico y evaluación de competencias. Barcelona, Graó.

Feliú Torruella, M. y Hernández Cardona, F.X. (2011). 12 ideas clave. Enseñar y aprender historia. Barcelona: Ed. Graó.

Gómez Carrasco, C. J., Miralles Martínez, P. (2013). La enseñanza de la Historia desde un enfoque social. Clío: History and History Teaching, 39.

Haydn, T., Arthur, J. y Hunt, M. (1997). Learning to teach History in the secondary schools. A companion to school experience. London/New York: Routledge.

Hernàndez Cardona, F.X. (2002). Didáctica de las ciencias sociales, geografía e historia. Barcelona: Ed. Graó.

Hernández Cervantes, L. (2014). La enseñanza y el aprendizaje del tiempo histórico en la educación infantil: Las representaciones sociales y la práctica docente de una estudiante de maestra de educación infantil. Enseñanza de las ciencias sociales: revista de investigación, 13.

Inarejos Muñoz, J. A. (2013). Nacionalismos e identidades en la enseñanza de la Historia de España: avances y resistencias. Clío: History and History Teaching, 39.

Miralles, P. y Molina, S. (2011). Didáctica de las ciencias sociales para el área de Conocimiento del entorno. En M.P. Rivero García (coord.), Didáctica de las ciencias sociales para Educación Infantil (89-110). Zaragoza: Ed. Mira.

Miralles, P. y Rivero, P. (2012). Propuestas de innovación para la enseñanza de la historia en Educación Infantil. Revista Electrónica Interuniversitaria de Formación del Profesorado 15, 81-90 (Consulta en 07/02/2017: http://www.aufop.com/aufop/uploaded_files/articulos/1335398854.pdf).

Molina Puche, S. (2011). El uso de la genealogía para la enseñanza de la Historia en los niveles educativos iniciales. Clío: History and History Teaching, 37.

Moradiellos, E. (2013). Clío y las aulas. Ensayo sobre Educación e Historia. Badajoz: Diputación de Badajoz.

Pagès Blanch, J. y Santisteban Fernández, A. (2010). La enseñanza y el aprendizaje del tiempo histórico en la Educación Primaria. Cedes 30, 82, 281-309. 
Pastor Blázquez, Ma M., Alonso Alberca, J. I. Luna Rodrigo, G., Jiménez-Ridruejo Gil, G. Martín Rodríguez Cerezo, T. y Santisteban Cimarro, A. (2015). Propuestas didácticas de carácter interdisciplinar para la enseñanza/aprendizaje del espacio y el tiempo en la educación infantil. Didácticas Específicas, 13, 87-104.

Prats, J. (2010), "En defensa de la Historia como materia educativa", en Tejuelo (2010), pp. 8-18.

Prieto, J.A, Gómez, C. J. y Miralles, P. (2013). El uso de fuentes primarias en el aula y el desarrollo del pensamiento histórico y social. Una experiencia en Bachillerato. Clío, 39.

Rodríguez Rodríguez, C.C. (2008). El protagonista de la semana. Cuadernos de pedagogía, 380 (Junio), 27-29.

Seixas, P. y Morton, T. (2013). The Big Six Historical Thinking Concepts. Toronto: Nelson.

Tonda Monllor, E.M. (2001). La didáctica de las ciencias sociales en la formación del profesorado en Educación Infantil. Alicante: Universidad de Alicante.

Trepat, C.A. (2002). El tiempo en la didáctica de las ciencias sociales. En C.A. Trepat y P. Comes, El tiempo en la didáctica de las ciencias sociales (pp. 7-122). Barcelona: Ed. Graó.

Trepat, C.A. (2011). El aprendizaje del tiempo en Educación Infantil. En M.P. Rivero García (coord.), Didáctica de las ciencias sociales para Educación Infantil (pp. 49-63). Zaragoza: Ed. Mira.

Trigueros Cano, J., Arias Ferrer, L., Miralles Martínez, P., Molina Puche, S. y Rodríguez Pérez, R. (2013). Ciencias Sociales y su didáctica. Educación Infantil. Murcia: CFDP Diego Marín. 